\title{
Clinical and immunological assessment in breast cancer patients receiving anticancer therapy and bovine dialyzable leukocyte extract as an adjuvant
}

\author{
HUMBERTO H. LARA* ${ }^{*}$ LILIANA IXTEPAN TURRENT**, ELSA N. GARZA-TREVIÑO \\ REYES TAMEZ-GUERRA and CRISTINA RODRIGUEZ-PADILLA \\ Laboratorio de Inmunología y Virología, Departamento de Microbiología e Inmunología, \\ Universidad Autonoma de Nuevo Leon, Nuevo Leon, Mexico
}

Received December 21, 2009; Accepted March 22, 2010

DOI: 10.3892/etm_00000066

\begin{abstract}
Dialyzable leukocyte extract (DLE) is one of the immunological agents used as an adjuvant in cancer therapy; it has been associated with improved quality of life during cancer chemotherapy. Based on these previous findings and on the observed clinical benefits attributed to DLE in other types of cancer, we investigated its clinical and immunological effects as a therapy adjuvant on breast cancer patients who received only chemotherapy, as compared to patients administered bovine DLE (bDLE) as an adjuvant. This study included 43 breast cancer patients who were about to begin chemotherapy. This group was divided as follows: 25 received chemotherapy and bDLE as an adjuvant therapy, and 18 received only chemotherapy without the adjuvant. All patient clinical and immunological responses were monitored. Among patients in the group that received bDLE as adjuvant, $60 \%$ showed a complete response, $32 \%$ showed a partial response and $8 \%$ did not respond. By contrast, in the group without the adjuvant, $39 \%$ showed a complete response, $50 \%$ displayed a partial response and $11 \%$ were non-responders. In addition, bDLE treatment in combination with chemotherapy resulted in the enhancement of the Karnofsky performance scale during chemotherapy. Even though patients underwent several cycles of chemotherapy without bDLE, the lymphocyte population dropped to below the reference value. On the other hand, in patients with bDLE as adjuvant, the $\mathrm{CD}^{+}$and $\mathrm{CD}^{+}{ }^{+}$lymphocytes and the B lymphocytes were maintained
\end{abstract}

Correspondence to: Dr Humberto H. Lara, Laboratorio de Inmunología y Virología, Departamento de Microbiología e Inmunología Universidad Autonoma de Nuevo Leon, Edificio C, 3er piso, Facultad de Ciencias Biologicas S/N, Ciudad Universitaria, C.P. 66451, San Nicolas de los Garza, Nuevo Leon, Mexico

E-mail: dr.lara.v@gmail.com

${ }^{*}$ Contributed equally

Key words: dialyzable leukocyte extract, immunotherapy agent, clinical response, Karnofsky performance scale within the median range of the reference value. The number of natural killer cells also increased after chemotherapy treatment with bDLE as an adjuvant. In conclusion, bDLE treatment contributes to significant immunological recovery in patients that have undergone heavy chemotherapy, increasing the clinical response and quality of life during chemotherapy.

\section{Introduction}

Breast cancer is the most common malignancy and the principal cause of cancer-related death among women globally (1). Statistics show that each year there are over 1.1 million women newly diagnosed with breast cancer worldwide. Each year 410,000 women die from the disease (2). The total cost of illness for breast cancer has been estimated at $\$ 3.8$ billion, of which $\$ 1.8$ billion represents medical care due to side effects during treatment. Among women who received chemotherapy this equated to more than $\$ 1,200$ in additional health care expenditures related to chemotherapy and more than $\$ 17,000$ in additional costs for ambulatory care as compared to women who did not receive chemotherapy (3).

Today, it is well known that anticancer treatment by surgery, radiotherapy or chemotherapy has improved the prognosis of the disease and has increased survival. In breast cancer, antineoplastic chemotherapy has improved the overall clinical response. The administration of taxane has increased the response rate from 50 to $68 \%$; with the combination of epirubicin and paclitaxel the overall response rate is $66 \%$ (4).

However, various side effects have been associated with chemotherapy and radiotherapy. These side effects, not only affect the tumor, but also target bone marrow activity and divide lymphocytes causing lymphocytopenia (5) which may induce subsequent clinical immunodeficiency (6). Chemotherapeutic drugs produce T-cell depletion, which is more severe in $\mathrm{CD}^{+}$than in $\mathrm{CD}^{+} \mathrm{T}$ lymphocytes, a decrease in the dendritic cell function and an alteration in the production of pro-inflammatory and anti-inflammatory cytokines.

Antineoplastic chemotherapy also induces side effects such as fatigue (7,8), skeletal muscle wasting and atrophy (9), as well as elevated levels of tumor necrosis factor, inactivity and weight loss. In 1948, Karnofsky developed a performance 
status scale as a multi-measure assessment of the quality of life for cancer patients during medical treatment $(10,11)$. Such investigations revealed that chemotherapy, not only generates medical benefits during the disease, but unfortunately also worsens the quality of life during treatment (12-15).

An improved immune response helps to prevent chemotherapy-induced side effects. An immunotherapy agent increases the populations of T-cells, dendritic and natural killer (NK) cells that are the most potent effectors in the host antitumor response. Immunotherapy agents are an alternative therapy used to boost antitumor immunity and to improve the clinical response to cancer chemotherapeutic treatment.

An immunological agent that has been considered in the context of cancer immunotherapy is the dialyzable leukocyte extract (DLE) or transfer factor, which has no reported side effects or toxicity. DLE was first described in 1955 by Lawrence and Borkowsky (16). In 1970, Kirkpatrick found that antigen-specific DLE therapy results in the induction of cell-mediated immunity and successful response to the corresponding antigen (17). Currently, DLE is defined as a dialyzed heterogeneous mixture of low molecular weight (<10 kDa) substances released from disintegrated blood or tissue leukocytes. DLE is believed to transfer the ability to express delayed-type hypersensitivity and cell-mediated immunity from an immune donor to a non-immune recipient (18). DLE has been used as a therapeutic agent in the treatment of autoimmune diseases (19), bacterial diseases (20), asthma and allergies (19) (Luna-Baca GA, Linares M, SantacruzValdes C, et al: Immunological study of patients with herpetic stromal keratitis treated with dialyzable leukocyte extracts. 13th International Congress of Immunology, 2007). Such treatment has consistently led to improved prognosis.

Therefore, DLE represents an attractive alternative to complement chemotherapy, which can be used to enhance the immune system after disturbances resulting from the side effects of chemotherapy. DLE in vitro is effective in improving cellular immunity (18) and in regulating the production of different cytokines involved in tumor progression (21-25).

In breast cancer cell line assays, bovine DLE (bDLE) induced cytotoxic effects despite suppressing the expression of p53 mRNA, bab-1, c-myc, bax, bcl-2 and bad mRNA $(26,27)$. In clinical trials, patients with advanced breast cancer were treated with pooled dialyzable transfer factor from healthy adult donors (non-specific) without chemotherapy or radiotherapy, after which the disease progressed $(21,28)$. In other reports, the administration of DLE directly to the tumor was found to reduce tumor size and increase CD2 ${ }^{+}, \mathrm{CD} 4^{+}, \mathrm{CD} 8^{+}$ and NK cell counts in rats with glioblastoma multiforme (29). DLE as an adjuvant of chemotherapy has been associated with tumor regression and temporary stabilization in several types of cancer (30), such as breast cancer, nasopharyngeal carcinoma (31), metastatic renal carcinoma (32), prostate cancer (33) and others (34).

Previously, we reported the use of bDLE as an adjuvant therapy to complement bevacizumab (Avastin), cetuximab (Erbitux), cytokines and cisplatin in transarterial chemoembolization (TACE). bDLE was shown to reduce tumor size in a lung cancer (stage III) patient and led to complete remission in 3 patients with primary pancreatic cancer (moderately differentiated). Furthermore, cellular immunity parameters were maintained within reference ranges after chemotherapy (Rodriguez-Padilla C, García de la Fuente A, Díaz R, et al: Intra-arterial chemo-inmuno target therapy plus conformal XRT in brain tumors. 16th International Congress on AntiCancer Treatment Paris, France, 2005) (Rodriguez-Padilla C, Ixtepan L, García de la Fuente A, et al: Transarterial chemoembolization (TACE) with bevacizumab (avastin), cetuximab (erbitux) and immunomodulators and imageguided radiation therapy (IGRT) in patients with lung cancer. 19th International Congress on Anti-Cancer Treatment Paris, France, 2008). The quality of life, as measured by the Karnofsky performance scale, increased.

Based on our previous experience with bDLE, the main objective of the present study was to assess the clinical and immune responses with regard to quality of life in breast cancer patients who were undergoing standard chemotherapy and who also received adjuvant therapy (bDLE).

\section{Patients and methods}

Patients. A total of 43 women with confirmed histological diagnoses of breast cancer were included in the study. Female patients over 18 years of age were seronegative for human immunodeficiency virus, human T-cell leukemia virus type 1 , hepatitis B and hepatitis C. Patients who were randomly selected for the treatment group had a Karnofsky performance status of $\geq 60 \%$. None of the patients received cell proliferation stimulants during chemotherapy [Neupogen or granulocyte colony-stimulating factor (G-CSF)], drugs to stimulate appetite or corticosteroids. The Institutional Review Board and Ethics Committee of the Universidad Autonoma de Nuevo Leon, Mexico approved the trial, and all patients gave their written informed consent.

The chemotherapy commonly employed for local disease includes doxorubicin and cyclophosphamide (AC), AC followed by paclitaxel, cyclophosphamide, doxorubicin and fluorouracil (FAC), cyclophosphamide, methotrexate, fluorouracil (CMF), docetaxel, doxorubicin and cyclophosphamide (TAC). For metastatic disease the regimens may also include epirubicin, Navelvine, Aromasin or Xeloda.

Adjuvant therapy. The bDLE used in our study as an adjuvant therapy in patients who received chemotherapy was produced by the Laboratory of Immunology and Virology at the Universidad Autonoma de Nuevo Leon, Mexico, following a modified protocol described by Lawrence and Borkowsky (16). bDLE is a mixture of low molecular weight molecules acquired from the dialyzation of disintegrated bovine spleens. The bDLE was lyophilized, tested for endogenous pyrogens using the Limulus amoebocyte lysate assay (MP Biomedicals Inc.) and determined to be free of bacterial contamination by culturing in different media as well as by in vivo mouse inoculations.

Study assessment. The design of the study included 43 breast cancer patients divided as follows: 25 breast cancer patients monitored for clinical and immunological responses during chemotherapy treatment with bDLE as adjuvant therapy and a control group that included 18 breast cancer patients receiving chemotherapy without bDLE as adjuvant. The administration of bDLE lasted 9 months, starting with 1-week 


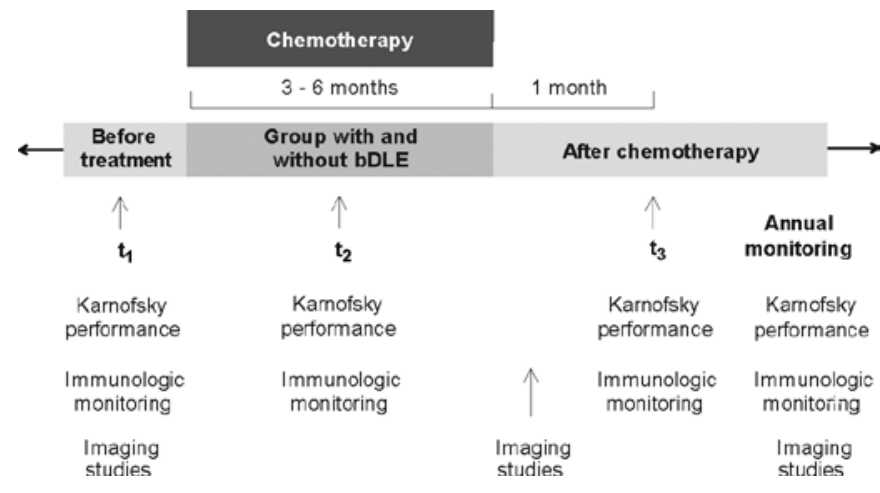

Figure 1. Timeline of the study design.

administration of bDLE alone prior to chemotherapy, with continued administration during the chemotherapy cycle (3-6 months) up to 1 month after the completion of chemotherapy. The dose administered to each patient was defined according to the patient's immunologic status. For the first 15 days, the daily administration of bDLE was as follows: i) $1-3 \%$ of B lymphocytes, 5 oral units; ii) 4-6\% of B lymphocytes, 4 units ( 2 oral $/ 2$ i.m.); iii) $>6 \%$ of B lymphocytes, 1 unit alternating oral and i.m. daily. All patients began the bDLE treatment before chemotherapy and continued with the daily treatment during all chemotherapy cycles and several months after the completion of the chemotherapy. If patients achieved a complete remission before 3 months with bDLE and chemotherapy, treatment was limited to bDLE until the follow-up appointment, at which point patients were evaluated immunologically based on their lymphocyte profiles.

Evaluation of the immunologic response. The immunologic parameters of the patients were monitored during chemotherapy in both groups. In addition, in the group that received bDLE as adjuvant the cellular immune response before receiving bDLE was evaluated also 1 month after finishing chemotherapy (description of the protocol design in Fig. 1). Monitoring involved obtaining complete and differential blood counts, as well as flow cytometric analysis of peripheral mononuclear cells. Flow cytometry was used to count NK cells, B lymphocytes and T lymphocytes. Flow cytometry was performed on a Beckman Coulter Altra No. AE47042. Data were obtained and analyzed using Software Expo 32 version 1.2.

bDLE stimulates an immune response mediated by cytokines that indirectly stimulate the proliferation of hematopoietic progenitor cells in bone marrow, as reported used pig-DLE in rats after radiotherapy (18). We evaluated several concentrations of IL-3 and IL-7 in serum with and without bDLE as an adjuvant during chemotherapy using an ELISA assay according to the protocol by Peprotech Company.

Evaluation of the clinical response. A total of 43 patients were evaluated for the clinical response to cancer chemotherapy treatment with or without bDLE as adjuvant, as determined by standard radiographic studies or PET-CT scan imaging. Clinical tumor response was compared to the control group (without bDLE) according to the International Union Against Cancer Criteria. A complete response (CR) was defined as the
Table I. Patient characteristics.

\begin{tabular}{|c|c|c|}
\hline Characteristics & With bDLE (\%) & Without bDLE (\%) \\
\hline Total patients, 43 & $25 \quad(60)$ & 18 (40) \\
\hline \multicolumn{3}{|l|}{ Pathological stage } \\
\hline $\mathrm{I}$ & $2 \quad(8)$ & 2 (11) \\
\hline II & $13(52)$ & $5 \quad(28)$ \\
\hline III & 4 (16) & $5 \quad(28)$ \\
\hline IV & $6 \quad(24)$ & $6 \quad(33)$ \\
\hline Total & $25(100)$ & $18(100)$ \\
\hline \multicolumn{3}{|l|}{ Tumor markers } \\
\hline $\mathrm{RE}$ & $13 \quad(52)$ & $10 \quad(56)$ \\
\hline $\mathrm{RP}$ & 8 (32) & 7 (39) \\
\hline Her2 & $7 \quad(28)$ & $6 \quad(33)$ \\
\hline Total & $25(100)$ & $18(100)$ \\
\hline \multicolumn{3}{|l|}{ Performance status } \\
\hline 0 & $1 \quad(4)$ & 2 (12) \\
\hline 1 & 24 (96) & $16(88)$ \\
\hline \multicolumn{3}{|l|}{ Clinical treatment } \\
\hline Surgery & $13(52)$ & $18(100)$ \\
\hline Chemotherapy & $25(100)$ & $18(100)$ \\
\hline Radiotherapy & $1 \quad(4)$ & 7 (39) \\
\hline Hormonal & $8 \quad(32)$ & $8 \quad(44)$ \\
\hline
\end{tabular}

disappearance of all clinical evidence of disease. A partial response (PR) was defined as a $\geq 50 \%$ decrease in the sum of the products of perpendicular diameters of all measurable lesions for at least 1 month with no increase in any lesion and no appearance of new lesions. Patients with mixed or minor responses or progressive disease were considered nonresponders (NR) (38).

Quality of life. Quality of life was measured in the group with and without bDLE using the Karnofsky performance scores before treatment with bDLE and after 1 month following the end of the chemotherapy regimen.

Statistical analysis. A t-test was used to compare lymphocyte cell populations and Karnofsky performance scores obtained before and after bDLE treatment. Statistical significance was established as $\mathrm{P}<0.05$. Individual values given in the figures represent the mean of 25 patients \pm SEM (for those who received adjuvant therapy) or 18 patients \pm SEM (for those who did not receive adjuvant therapy).

\section{Results}

Patient characteristics. To establish a general screening assessment of the effect of bDLE as an adjuvant during chemotherapy, 25 patients with a diagnosis of breast cancer were selected randomly. The study also included 18 breast cancer patients who did not receive adjuvant treatment with bDLE during chemotherapy. In both groups, the patients who had disseminated metastasis were affected in a median of 
Table II. Effect of bDLE treatment on the cellular immune response.

\begin{tabular}{lccccc}
\hline Treatment & Leukocytes & CD4 & CD8 & CD19 & CD56-16 $^{+}$ \\
\hline Before bDLE & $5,928 \pm 339$ & $713 \pm 50$ & $458 \pm 46$ & $123 \pm 17$ & $157 \pm 27$ \\
After bDLE & $5,554 \pm 374$ & $877 \pm 95$ & $475 \pm 102$ & $314 \pm 69$ & $271 \pm 48$ \\
P-value & 0.05 & 0.05 & 0.05 & $<0.05$ & $<0.05$ \\
\hline
\end{tabular}

Table III. Clinical responses to treatment.

\begin{tabular}{|c|c|c|c|c|c|}
\hline Stage & Treatment & Complete response $(\%)$ & Partial response $(\%)$ & No response $(\%)$ & Overall response for stage $(\%)$ \\
\hline \multirow[t]{2}{*}{ I } & With bDLE & $2(100)$ & 0 & 0 & 100 \\
\hline & Without bDLE & $2(100)$ & 0 & 0 & \\
\hline \multirow[t]{2}{*}{ II } & With bDLE & $9 \quad(70)$ & $4(30)$ & 0 & 72 \\
\hline & Without bDLE & $4 \quad(80)$ & $1(20)$ & 0 & \\
\hline \multirow[t]{2}{*}{ III } & With bDLE & $2(50)$ & $2(50)$ & 0 & 33 \\
\hline & Without bDLE & 1 (20) & $3(60)$ & $1(20)$ & \\
\hline \multirow[t]{2}{*}{ IV } & With bDLE & $2(33)$ & $2(33)$ & $2(33)$ & 17 \\
\hline & Without bDLE & 0 & $5(83)$ & $1(17)$ & \\
\hline
\end{tabular}
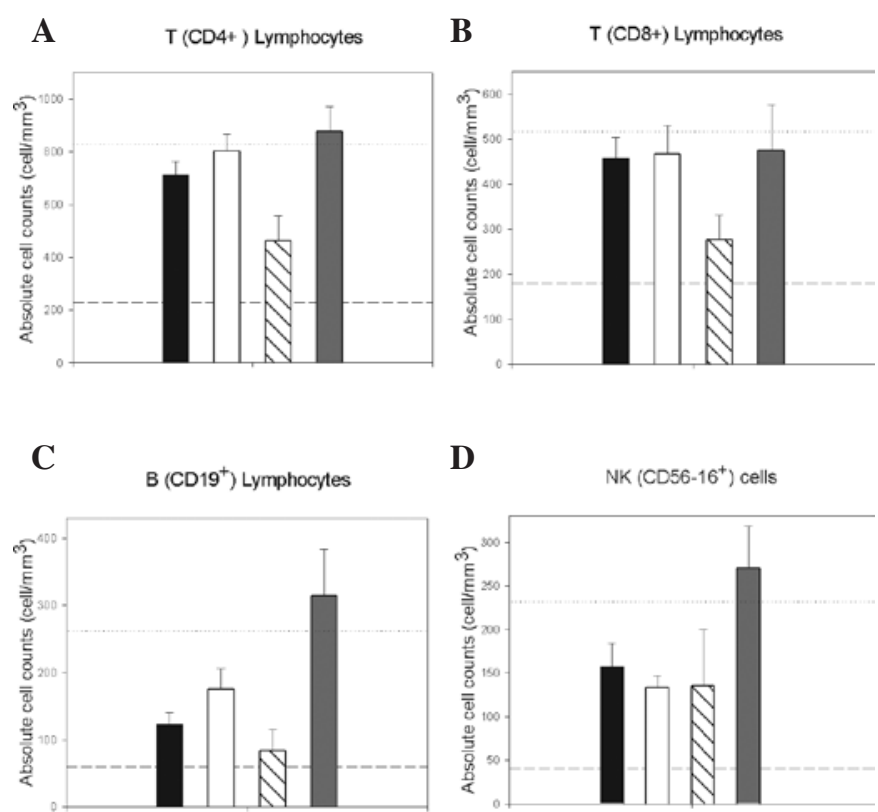

Before chemotherapy During chemotherapy vith bDLE Minimum reference value for a standard population
D

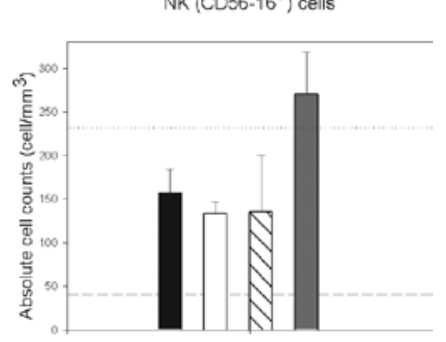
Median of reference range value for a standard population After chemotherapy with bDLE

Figure 2. Effect of bDLE treatment on the cellular immune response. Absolute cell counts of $\mathrm{T}\left(\mathrm{CD}^{+}\right)$lymphocytes, $\mathrm{T}\left(\mathrm{CD} 8^{+}\right)$lymphocytes, $\mathrm{B}$ $\left(\mathrm{CD} 19^{+}\right)$lymphocytes and NK $\left(\mathrm{CD}^{-1} 6^{-16^{+}}\right)$cells: reference values (without chemotherapy) during bDLE and chemotherapy and after chemotherapy.

three organs/tissues, principal bones, liver and lung. Among patients who received bDLE during chemotherapy, $84 \%$ were positive for tumor markers. In the control group, $88 \%$ were

positive for tumor markers. According to Karnofsky performance scale classification, 0 patients were able to work and 1 patient was not. All patients received a tailored oncology treatment scheme depending on disease stage (Table I).

Immunologic response. The median total white blood cell count before chemotherapy was $5,928 \pm 339 / \mathrm{mm}^{3}$. During chemotherapy, this measure was slightly reduced in the group that received bDLE as adjuvant to $5,554 \pm 374 / \mathrm{mm}^{3}$ and in the control group to $4,779 \pm 435 / \mathrm{mm}^{3}$. The percentages of monocytes, basophils, eosinophils and neutrophils were always reported to be in the reference range values for our laboratory, even during chemotherapy treatment.

No myelosuppression in lymphoid populations was observed in patients receiving the bDLE treatment, while in patients undergoing chemotherapy without bDLE the absolute numbers of $\mathrm{CD}^{+}, \mathrm{CD}^{+}$and $\mathrm{B}$ lymphocytes were reduced compared to the reference range values as shown in Fig. 2. Interestingly, a significant increase in the numbers of $\mathrm{NK}$ cells $(\mathrm{P}<0.05)$ and $\mathrm{B}$ lymphocytes $(\mathrm{P}<0.05)$ was observed 1 month after the completion of chemotherapy in patients receiving bDLE as an adjuvant (Table II). The proportion of lymphocytes was maintained at reference values during treatment with bDLE as adjuvant. Levels in the control group were below reference values as reported by Mackall et al for patients undergoing chemotherapy (5).

In addition, IL-3 levels were reduced by $80 \%$ in the group that received chemotherapy without bDLE. In the group with bDLE as adjuvant during chemotherapy we observed that levels were reduced by only $34 \%$. However, IL-7 levels were increased in the group that received bDLE as adjuvant $(11 \%)$ prior to chemotherapy in combination with bDLE. This measure was reduced by $30 \%$ in the control group during chemotherapy as compared to before chemotherapy (Fig. 3). 
A

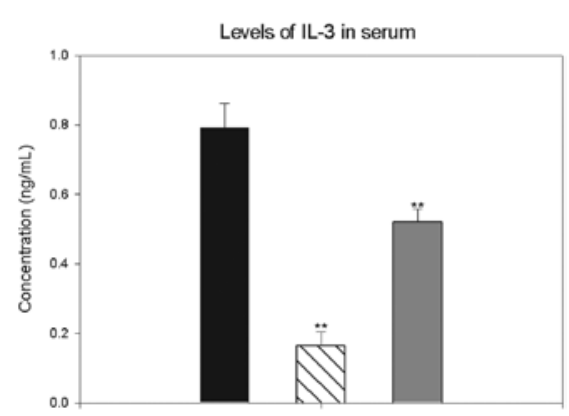

B

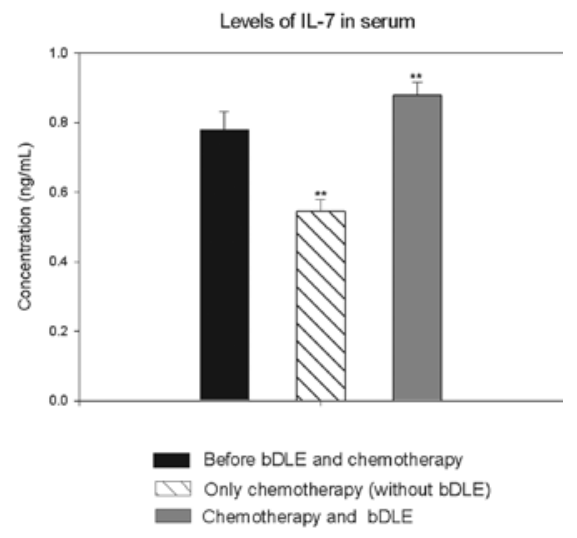

Figure 3. Concentration of cytokines in the serum of breast cancer patients with and without bDLE as an adjuvant to chemotherapy. (A) Levels of IL-3 with and without bDLE during chemotherapy. (B) Levels of IL-7 in patients with and without bDLE during chemotherapy.

Clinical response. Clinical response was evaluated using standard radiographic studies or PET-CT scan imaging.

In the group with bDLE as adjuvant therapy, 15 (60\%) patients experienced a CR to treatment, 8 (32\%) experienced a PR and $2(8 \%)$ were NRs (Table III). Among stage I patients in this group, 2 (100\%) patients experienced a complete response (CR); among stage II patients, $9(70 \%)$ experienced a CR and $4(30 \%)$ patients experienced a PR; for stage III patients, $2(50 \%)$ experienced a CR and $2(50 \%)$ patients experienced a PR; among stage IV patients, 2 (33\%) experienced a CR, 2 (33\%) experienced a PR and $2(33 \%)$ patients experienced NR.

In the control group (without adjuvant treatment with bDLE during chemotherapy), 7 (39\%) patients experienced a CR to treatment (chemotherapy or radiotherapy), 9 (50\%) experienced a PR and 2 (11\%) were NRs (Table III). Among stage I patients in the control group, $2(100 \%)$ patients experienced a CR; for stage II, $4(80 \%)$ patients experienced a CR and $1(20 \%)$ patient experienced a PR; among stage III patients, 1 (20\%) experienced a CR, $3(60 \%)$ experienced a PR and $1(20 \%)$ patient exhibited NR; among stage IV patients, none experienced a CR, $5(83 \%)$ patients experienced a PR (17\%) and 1 patient experienced NR.

PET-CT imaging. We used PET-CT to evaluate both groups and observed that in the group with bDLE as adjuvant the regression of metastatic lesions in diverse anatomic locations was obtained in less time than in the control group (without bDLE). As shown in Fig. 4A, patients with metastatic breast cancer without adjuvant therapy had persistent thyroid lesions
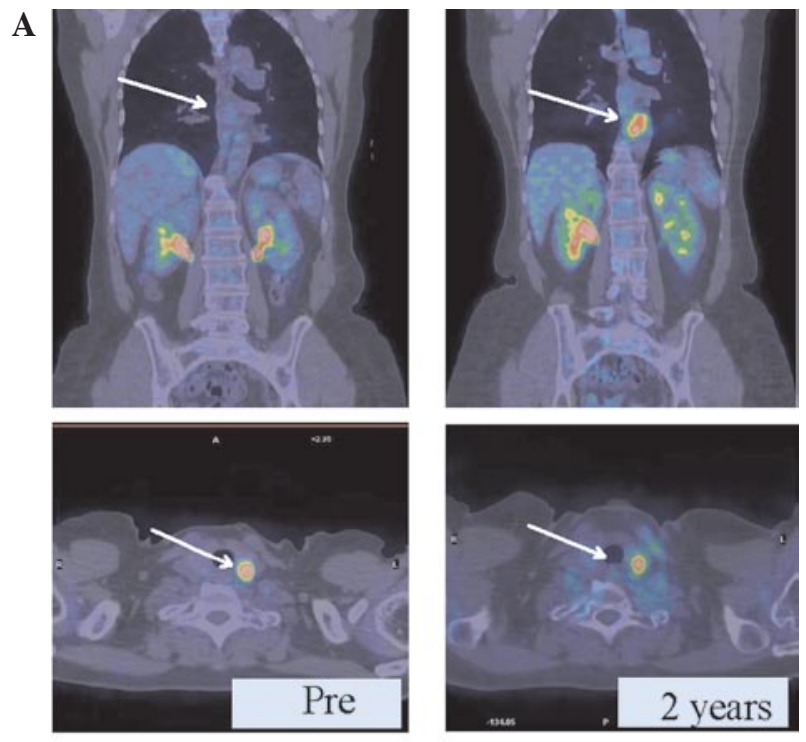

B
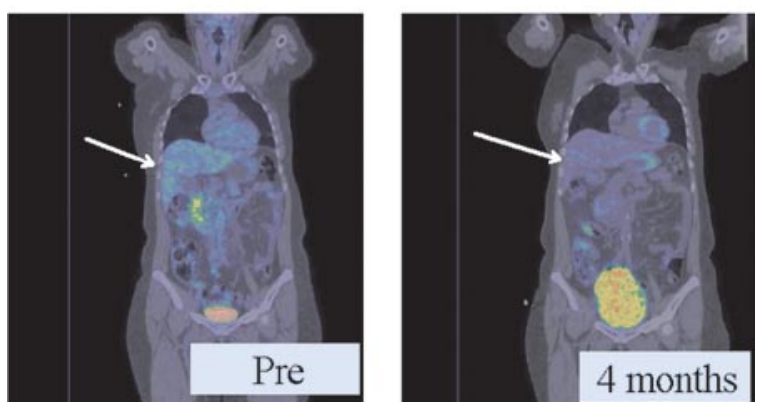

Figure 4. Clinical effect of bDLE as visible in PET images. In the control group, we observed no response (A) of thyroid lesions after chemotherapy without bDLE. (B) Partial regression of retroperitoneal retrohepatic metastases after chemotherapy and bDLE treatment. Pre-, pre-treatment.

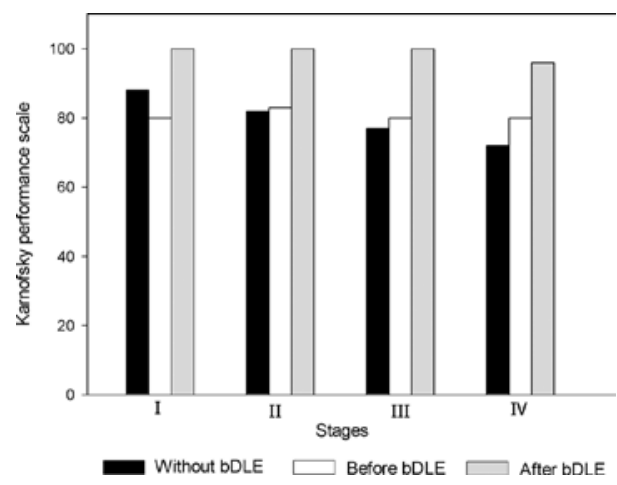

Figure 5. Karnofsky performance scale by stage without bDLE during chemotherapy and with bDLE treatment before and during chemotherapy.

and a new lesion around the aorta $(2 \mathrm{~cm})$ after 2 years of chemotherapy. In another case (Fig. 4B), retroperitoneal retrohepatic metastases exhibited a PR, with the same metabolic activity (6 SUV) after only 4 months of receiving bDLE treatment and 5 cycles of chemotherapy.

Quality of life. Quality of life was measured using the Karnofsky performance scores. In the patients who received bDLE adjuvant therapy during chemotherapy, average Karnofsky scores increased from 70 to 90 , which reflected 
an overall clinical improvement in the health status of the patients (Fig. 5). Of the patients who received chemotherapy treatment without bDLE adjuvant therapy, the average final score was 80 .

Toxicity. The administration of bDLE therapy was safe and well tolerated. None of the patients died during the reported period.

\section{Discussion}

DLE, commonly known as transfer factor, is an immunotherapy agent that has been reported to improve the immunological response in cancer patients (Rodriguez-Padilla C, García de la Fuente A, Díaz R, et al: Intra-arterial chemo inmuno target therapy plus conformal XRT in brain tumors. 16th International Congress on Anti-Cancer Treatment Paris, France, 2005) (36). Various reports have used different clinical assays to investigate DLE as an adjuvant therapy. These studies have consistently reported improvement in the clinical response to treatment, but there is a lack of information about the clinical parameters that are improved in those patients (37-39). In this clinical study, we randomly sampled breast cancer patients to explore the immunological and clinical response to bDLE treatment as an adjuvant to chemotherapy. In particular, we focused on the clinical effects of bDLE as an adjuvant therapy during chemotherapy.

Myelosuppression is a common side effect of chemotherapy that is accompanied by lymphopenia, neutropenia and thrombocytopenia. (6). In this study, our results showed a protective effect of bDLE on $\mathrm{CD}^{+}{ }^{+} \mathrm{T}$ lymphocytes, $\mathrm{CD} 8^{+}$ T lymphocytes, CD19+ B lymphocytes and NK cells (Fig. 2). The absolute numbers of these lymphocytes in the bDLEtreated patients during chemotherapy (Fig. 2) were always higher than expected as compared to our control group and as reported by Mackall et al for patients undergoing chemotherapy (5). In addition, we observed that the levels of IL-3 and IL-7 were higher in the group that received bDLE as an adjuvant during chemotherapy as compared to the control group.

These factors likely underline the immunological protection afforded by bDLE during chemotherapy as reported by Vacek et al (18) using pig-DLE.

The administration of bDLE in this study resulted in an increased clinical response. The difference was principally observed in stage III and IV patients. The median survival reported after the appearance of metastases is approximately 20-25 months, hence the importance of obtaining a clinical response as rapidly as possible. We observed that those metastatic patients receiving bDLE exhibited improved clinical responses in 6-12 months, as compared to the group that did not receive adjuvant therapy with bDLE. In the latter group, the clinical response was as expected at approximately 2-3 years (data not shown).

Therefore, in future studies with bDLE as adjuvant chemotherapy, it will be necessary to focus specifically on the group that improved (patients with metastatic disease). To further verify enhancement due to bDLE treatment, we recommend a study with a larger population.

bDLE treatment in combination with chemotherapy resulted in an increase in the Karnofsky performance scores after several chemotherapy cycles; patients reached a 90 on the Karnofsky performance scores, which implies minor symptoms and the ability to work (10). By contrast, the average score for the control group (without bDLE) was 80 . During the interviews, we observed that patients improved in their general health and state of mind even 1 month after chemotherapy. Therefore, adjuvant therapy with bDLE reduces economic losses as well as the physical incapacitation suffered by cancer patients.

In conclusion, our results pertaining to the administration of bDLE as an adjuvant therapy during breast cancer chemotherapy can be used for clinical decision-making and for improving the quality of life during treatment. We propose the use of bDLE as an adjuvant to complement conventional chemotherapies in cancer. bDLE would be particularly useful to improve immunological response, symptomatology and general patient prognosis.

\section{Acknowledgements}

This is the first publication to report a clinical approach to define the anticancer effects of bDLE in breast cancer patients. The following funding source supported the data collection process: the Programa de Apoyo a la Investigacion en Ciencia y Tecnologia (PAICyT) from the Universidad Autonoma de Nuevo Leon, Mexico and the Consejo Nacional de Ciencia y Tecnologia (CONACyT), Mexico.

\section{References}

1. Bray F, McCarron $\mathrm{P}$ and Parkin DM: The changing global patterns of female breast cancer incidence and mortality. Breast Cancer Res 6: 229-239, 2004

2. Stewart BW and Coates AS: Cancer prevention: a global perspective. J Clin Oncol 23: 392-403, 2005.

3. Smith MD and McGhan WF: Financial facts about treating breast cancer. Bus Health 14: 67-68; 70, 1996.

4. Spitler LE: Clinical uses of transfer factor. Calif Med 118: 47, 1973.

5. Mackall CL, Fleisher TA, Brown MR, Magrath IT, Shad AT, Horowitz ME, Wexler LH, Adde MA, McClure LL and Gress RE: Lymphocyte depletion during treatment with intensive chemotherapy for cancer. Blood 84: 2221-2228, 1994.

6. Van der Most RG, Currie AJ, Robinson BW and Lake RA: Decoding dangerous death: how cytotoxic chemotherapy invokes inflammation, immunity or nothing at all. Cell Death Differ 15: 13-20, 2008.

7. Piper BF, Borneman T, Sun VC, Koczywas M, Uman G, Ferrell B and James RL: Cancer-related fatigue: role of oncology nurses in translating National Comprehensive Cancer Network Assessment Guidelines into practice. Clin J Oncol Nurs 12: 37-47, 2008.

8. Berger AM: Patterns of fatigue and activity and rest during adjuvant breast cancer chemotherapy. Oncol Nurs Forum 25: 51-62, 1998.

9. Goodman MN: Tumor necrosis factor induces skeletal muscle protein breakdown in rats. Am J Physiol 260: E727-E730, 1991.

10. Karnofsky DA, Abelmann WH and Craver LF: The use of the nitrogen mustards in palliative treatment of carcinoma. Cancer 1: 634-656, 1948.

11. Priestman TJ and Baum M: Evaluation of quality of life in patients receiving treatment for advanced breast cancer. Lancet 1: 899-900, 1976.

12. Kasper CE: Sarcolemmal disruption in reloaded atrophic skeletal muscle. J Appl Physiol 79: 607-614, 1995.

13. Kasper CE: Recovery of plantaris muscle from impaired physical mobility. Biol Res Nurs 1: 4-11, 1999.

14. Kasper CE and Sarna LP: Influence of adjuvant chemotherapy on skeletal muscle and fatigue in women with breast cancer. Biol Res Nurs 2: 133-139, 2000. 
15. St Pierre BA, Kasper CE and Lindsey AM: Fatigue mechanisms in patients with cancer: effects of tumor necrosis factor and exercise on skeletal muscle. Oncol Nurs Forum 19: 419-425, 1992.

16. Lawrence HS and Borkowsky W: Transfer factor - current status and future prospects. Biotherapy 9: 1-5, 1996.

17. Kirkpatrick $\mathrm{CH}$ : Transfer of cellular immunity with transfer factor. J Allergy Clin Immunol 63: 71-73, 1979.

18. Vacek A, Hofer M, Schneiderova H and Svoboda J: Ultrafiltered pig leukocyte extract (UPLE, IMUNOR) potentiates hematopoiesis-stimulating effects of G-CSF in vitro and improves the outcome of treatment of hematopoietic radiation damage in mice with G-CSF. Immunopharmacol Immunotoxicol 27: 647-659, 2005.

19. Pizzo PA, Henderson ES and Leventhal BG: Acute myelogenous leukemia in children: a preliminary report of combination chemotherapy. J Pediatr 88: 125-130, 1976.

20. Franco-Molina MA, Mendoza-Gamboa E, Castillo-Leon L, Tamez-Guerra RS and Rodriguez-Padilla C: Bovine dialyzable leukocyte extract protects against LPS-induced, murine endotoxic shock. Int Immunopharmacol 4: 1577-1586, 2004.

21. Atzpodien J, Kuchler T, Wandert $\mathrm{T}$ and Reitz M: Rapid deterioration in quality of life during interleukin-2- and alphainterferon-based home therapy of renal cell carcinoma is associated with a good outcome. Br J Cancer 89: 50-54, 2003.

22. Ojeda MO, van't Veer C, Fernandez Ortega CB, Arana Rosainz MJ and Buurman WA: Dialyzable leukocyte extract differentially regulates the production of TNFalpha, IL-6 and IL-8 in bacterial component-activated leukocytes and endothelial cells. Inflamm Res 54: 74-81, 2005.

23. Kirkpatrick $\mathrm{CH}$, Rozzo SJ, Mascali JJ and Merryman CF Murine transfer factor. II. Transfer of delayed hypersensitivity to synthetic antigens. J Immunol 134: 1723-1727, 1985.

24. Kirkpatrick $\mathrm{CH}$ : Therapeutic potential of transfer factor. N Engl J Med 303: 390-391, 1980.

25. Kirkpatrick $\mathrm{CH}$ : Transfer of delayed cutaneous hypersensitivity with transfer factor. Cell Immunol 41: 62-71, 1978

26. Franco-MolinaMA, Mendoza-GamboaE, Miranda-HernandezD, Zapata-Benavides P, Castillo-Leon L, Isaza-Brando C, Tamez-Guerra RS and Rodriguez-Padilla C: In vitro effects of bovine dialyzable leukocyte extract (BDLE) in cancer cells. Cytotherapy 8: 408-414, 2006.

27. Mendoza-Gamboa E, Franco-Molina MA, Zapata-Benavides P, Castillo-Tello P, Vera-Garcia ME, Tamez-Guerra RS and Rodriguez-Padilla C: Bovine dialyzable leukocyte extract modulates AP-1 DNA-binding activity and nuclear transcription factor expression in MCF-7 breast cancer cells. Cytotherapy 10: 212-219, 2008
28. Oettgen HF, Old LJ, Farrow JH, Valentine FT, Lawrence HS and Thomas L: Effects of dialyzable transfer factor in patients with breast cancer. Proc Natl Acad Sci USA 71: 2319-2323, 1974.

29. Pineda B, Estrada-Parra S, Pedraza-Medina B, RodriguezRopon A, Perez R and Arrieta O: Interstitial transfer factor as adjuvant immunotherapy for experimental glioma. J Exp Clin Cancer Res 24: 575-583, 2005.

30. Meier CR and LoBuglio AF: Transfer factor: a potential agent for immunotherapy of cancer. World J Surg 1: 617-623, 1977.

31. Goldenberg GJ and Brandes LJ: In vivo and in vitro studies of immunotherapy of nasopharyngeal carcinoma with transfer factor. Cancer Res 36: 720-723, 1976.

32. Moss RW: Cancer and complementary and alternative medicine in Italy: personal observations and historical considerations. Integr Cancer Ther 3: 173-188, 2004.

33. Pizza G, De VC, Cuzzocrea D, Menniti D, Aiello E, Maver P, Corrado G, Romagnoli P, Dragoni E and LoConte G: A preliminary report on the use of transfer factor for treating stage D3 hormone-unresponsive metastatic prostate cancer. Biotherapy 9 : $123-132,1996$.

34. Rodriguez-Flores A, Castillo-Juarez P, Vazquez D, Gonzalez-Guzman R, Rico-Martinez G and Estrada-Parra S Immunological evaluation of patients with osteosarcoma received adjuvant treatment with specific transfer factor. J Immunol 182: 63, 2009.

35. Dudley ME, Wunderlich JR, Yang JC, Sherry RM, Topalian SL, Restifo NP, Royal RE, Kammula U, White DE and Mavroukakis SA: Adoptive cell transfer therapy following non-myeloablative but lymphodepleting chemotherapy for the treatment of patients with refractory metastatic melanoma. J Clin Oncol 23: 2346-2357, 2005.

36. Franco-Molina MA, Mendoza-Gamboa E, Zapata-Benavides P, Vera-Garcia ME, Castillo-Tello P, Garcia dlF, Mendoza RD, Garza RG, Tamez-Guerra RS and Rodriguez-Padilla C: IMMUNEPOTENT CRP (bovine dialyzable leukocyte extract) adjuvant immunotherapy: a phase I study in non-small cell lung cancer patients. Cytotherapy 10: 490-496, 2008.

37. Fujisawa T: Transfer factor immunotherapy as an adjunct to surgery in lung cancer. Nihon Kyobu Shikkan Gakkai Zasshi 23: 68-73, 1985.

38. Krown SE, Pinsky CM, Hirshaut Y, Hansen JA and Oettgen HF: Effects of transfer factor in patients with advanced cancer. Isr J Med Sci 14: 1026-1038, 1978.

39. Vacek A, Hofer M, Hola J, Weiterova L, Streitova D and Svoboda J: The role of G-CSF and IL-6 in the granulopoiesisstimulating activity of murine blood serum induced by perorally administered ultrafiltered pig leukocyte extract, IMUNOR. Int Immunopharmacol 7: 656-661, 2007. 